\title{
Segmental absence of intestinal muscle with ileal web in an extremely low birth weight infant. Case report
}

\author{
Mehmet Buyuktiryaki, M.D. ${ }^{a}$, Hayriye G. Kanmaz, M.D. ${ }^{a}$, Nilüfer Okur, M.D. ${ }^{a}$, Ufuk Ates, M.D. ${ }^{b}$, \\ Ali L. Sirvan, M.D. ${ }^{c}$ and Nurdan Uras, M.D. ${ }^{a}$
}

\begin{abstract}
Spontaneous intestinal perforations are localized perforations without the typical clinical, radiological, and histopathological features of necrotizing enterocolitis. Spontaneous intestinal perforation is a recently defined clinical entity. The best-known risk factor is prematurity. It is seen $2-3 \%$ in very low birthweight infants and $5 \%$ of extremely low birthweight infants. Herein we report an extremely low birthweight infant with spontaneous intestinal perforation, segmental absence of intestinal muscle and an ileal web as an underlying cause. We aimed to draw attention to the segmental absence of intestinal muscle which is rare but increasingly reported cause of spontaneous intestinal perforation and theimportance of histopathologic examination of surgical specimens.

Key words: spontaneous intestinal perforation, preterm infant, intestinal muscle, ileal web.
\end{abstract}

http:/ / dx.doi.org/10.5546/aap.2016.eng.e108

\section{INTRODUCTION}

A spontaneous intestinal perforation (SIP) is a single intestinal perforation typically involving the antimesenteric border of distal ileum, which usually occurs without a defined prodrome in extremely premature infants in the first 1-2 weeks of life. Its incidence is $2-3 \%$ in very low birthweight (VLBW) infants and 5\% in extremely low birthweight infants (ELBW). ${ }^{1}$ The best known risk factor is prematurity. ${ }^{2}$ An intestinal web is a rare congenital anomaly and typically

a. Division of Neonatology, Zekai Tahir Burak Maternity Teaching Hospital, Ankara, Turkey.

b. Division of Pediatric Surgery, Zekai Tahir Burak Maternity Teaching Hospital, Ankara, Turkey.

c Division of Pathology, Zekai Tahir Burak Maternity Teaching Hospital, Ankara, Turkey.

E-mail Address:

Dr. Mehmet Buyuktiryaki: mbuyuktiryaki@yahoo.com

Funding: none.

Conflict of interest: none.

Received: 8-3-2015

Accepted: 10-15-2015 reported in childhood. ${ }^{3}$ Likewise, segmental absence of the intestinal muscle (SAIM) is also a rare condition and its pathogenesis has not been completely elucidated. There are usually intestinal perforation or obstruction symptoms. The lack of muscles in any part of the intestinal system leads to obstruction due to the absence of peristaltic movements and dilatation of the affected segment. It is not clear regarding the pathogenesis, whether perfortion is primarily due to the malformation or is secondary to ischemia and inflammation. Ischemia and hypoxia are the most important etiological possibilities as most cases are seen in infants with respiratory distress syndrome (RDS) and very low birthweight.

Herein we present an ELBW infant with RDS who underwent surgery for spontaneous intestinal perforation due to segmental absence of intestinal muscle accompanied by an ileal web.

\section{CASE}

A male infant with a birth weight of $815 \mathrm{~g}$ and gestational age of $24^{5 / 7}$ weeks was delivered through the spontaneous vaginal route following the first pregnancy of a 23-year-old mother. The mother had not adequate prenatal care and was admitted to the emergency department with contractions and preterm labor. Emergency obstetric ultrasound screening revealed polyhydramniosis; otherwise the infant was healthy. The mother gave birth soon after her admission. Neonatal resuscitation and intubation was performed in the delivery room. The Apgar score was 3 at the 1 st minute and 7 at the 5 th minute. At the admission to the neonatal intensive care unit, vital signs were: heart rate 145/minute, blood pressure 57/39 (45) $\mathrm{mmHg}$, saturation $94 \%$. Surfactant $(100 \mathrm{mg} / \mathrm{kg}$ beractant) was administered for clinical and radiologic findings of respiratory distress syndrome. Umbilical vein and artery catheters were inserted. A loading dose of intravenous caffeine $(20 \mathrm{mg} / \mathrm{kg}$ ) was administered; the maintenance dose was $5 \mathrm{mg} /$ $\mathrm{kg} /$ day. He passed meconium on $42 \mathrm{~h}$ of life and bowel sounds were audible; enteral nutrition was initiated $(15 \mathrm{cc} / \mathrm{kg} /$ day) with breast milk. 
Bile-containing residues were observed at 70 $\mathrm{h}$ of life. Plain abdominal radiograph revealed dilated bowel loops but no free air, air-fluid level or pneumatosis intestinalis. Withdrawal of enteral feedings and orogastric decompression was performed. The patient was doing well otherwise the feeding intolerance. On the $5^{\text {th }}$ day of life a sudden and significant abdominal distension with bluish color change on the abdominal skin occurred. Free air under the diaphragm was observed on plain abdominal radiograph, erected position. Empirical antibiotic therapy was administered since nosocomial sepsis could not be excluded.

The general condition was poor with hyperglycemia, acute renal failure and hemodynamic instability. A primary peritoneal drainage (PPD) was performed. The clinical condition was better on the postnatal 8th day with no discharge from the orogastric catheter or the drain. The drain was removed as bowel movement was present, spontaneous defecation occurred and the patient was hemodynamically stable. However, on the $11^{\text {th }}$ day abdominal distension developed and free air under the diaphragm was detected on abdominal graph, thus the case underwent to surgery. A $20 \mathrm{~cm}$ collapsed ileal segment was found proximal to the cecum. There were also two ileum perforations located at 30 and 40 centimeters proximal to the cecum. Antimesenteric wedge resection of the perforated segment and end-to-end anastomosis

FIgURE 1. Perforation of the ileum

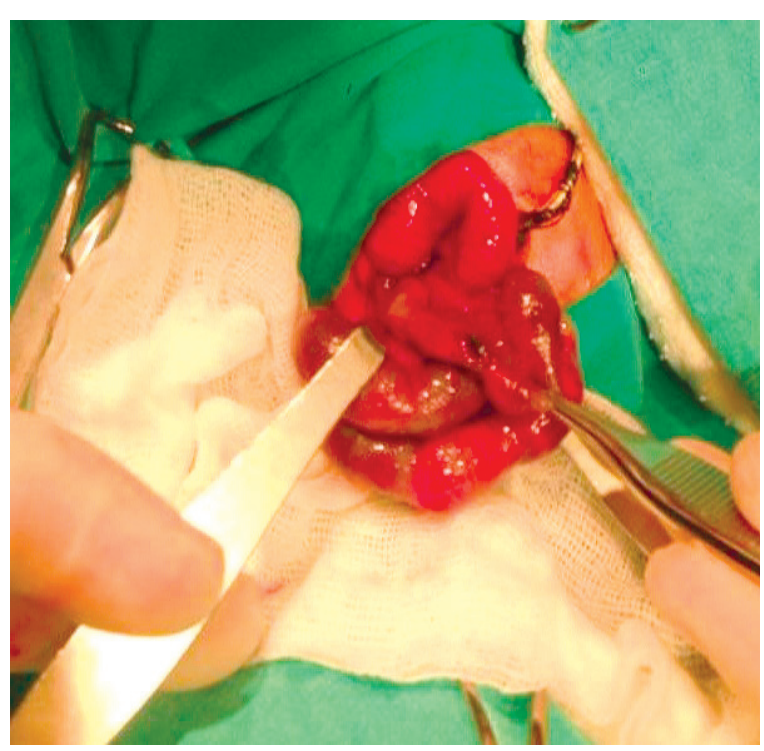

were performed. The bowel was observed to be macroscopically normal except the two fore mentioned perforations (Figure 1). The border of the normal and collapsed ileum was excised and an $8 \mathrm{Fr}$ feeding probe was introduced through the collapsed ileum and an intestinal web was found $5 \mathrm{~cm}$ distally. A $6 \mathrm{~cm}$ ileum segment was resected together with the web. After resection, the remaining two ends of the ileum were anastomosed. The histopathological examination of the resected ileal segments revealed normal intestinal mucosa and edematous submucosa with slightly infiltration of lymphocytes along with segmental absence of intestinal muscle (Figure 2). The serosa was normal and there was no necrosis. The patient died on the postnatal 15th day due to sepsis and multi-organ failure. Parents did not give consent thus autopsy could not be performed.

\section{DISCUSSION}

The etiology of SIP remains unknown. Although thinning or absence of the muscularis propria at the perforation site has been reported previously as similar to our case, it is unclear whether these changes are involved in the pathogenesis of SIP. Furthermore an ileal web was found in our case. Both pathologies may be caused by a vascular kinking in utero that leads to decreased intestinal perfusion and ischemia of the respective segment of bowel and make the intestines susceptible to perforation. ${ }^{4,5}$
FIGURE 2. Intact mucosa and edematous submucosa with slightly infiltration of lymphocytes along with segmental absence of muscle layer (HEE 20X)

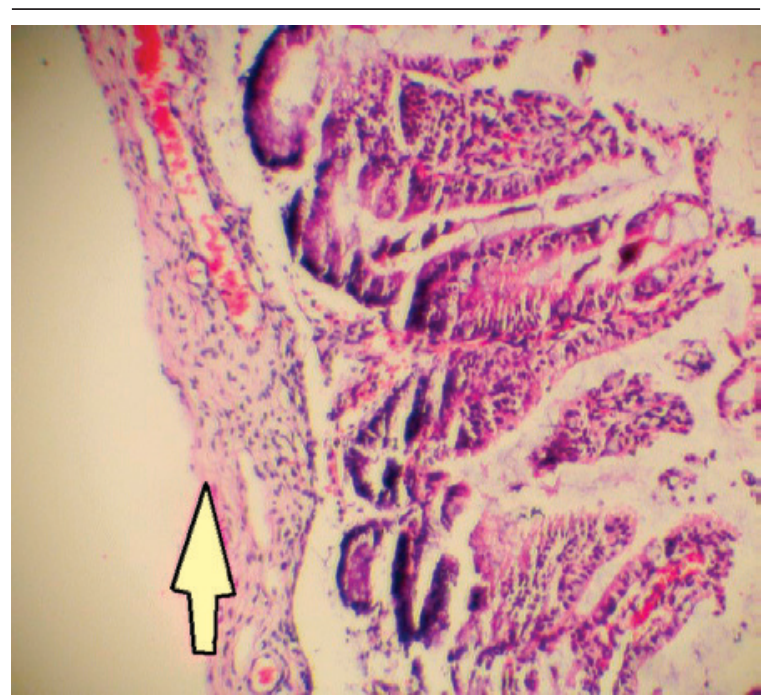


Several SIP cases that have abnormal intestinal muscle in the histopathological examination were reported in the literature. ${ }^{6}$ To our knowledge this is the first case in the literature that has both SAIM and ileal web as an underlying cause of SIP. Many authors emphasized that the lack of muscle was not a congenital malformation but secondary to ischemic damage however it is not clear yet why the mucosa, which is more vulnerable to ischemia than muscle, remains intact. ${ }^{7}$ It is a known fact that the regeneration capacity of mucosa and muscles is different so that the mucosa layer is regenerated following ischemia but the muscular layer cannot be regenerated and there is isolated lack of muscle. We can speculate that the web was formed as a result of exaggerated regeneration response to ischemia. ${ }^{6}$

SAIM presents with obstruction symptoms; polyhidramniosis which is reported in this case could suggest in-utero intestinal obstruction despite dilated bowel loops or increased echogenicity were not seen in antenatal ultrasonography. Presence of normal bowel sounds with gas distribution extending to the rectum on plain radiograph and spontaneous defecation in the first 3 days life suggested a partial obstruction and was the cause of atypical presentation. Segmental absence of the intestinal muscle is an uncommon clinical entity; however, its clinical presentation and progression is similar to more common presentations of peritonitis. Exploratory laparotomy is the gold standard in these clinical scenarios, and diagnosis depends on the histopathology of resected specimens. ${ }^{8}$ Primary peritoneal drainage is being increasingly used as initial treatment of SIP in NICU's since it is performed at the bedside and have comparable outcomes with exploratory laparotomy. 9,10 However, there will be no chance to get specimens from the intestine and rare underlying causes such as SAIM could not be defined in this technique.

Segmental absence of intestinal muscle represents a distinct entity, which may present in a variety of ways. This is the first report that describes its association with ileal web and may support the theory that proposes as etiology, a focal intrauterine ischemia of the developing gastrointestinal tract. Consideration should be given to biopsy and pathologic evaluation at the time of surgery.

\section{REFERENCES}

1. Meyer CL, Payne NR, Roback SA. Spontaneous, isolated intestinal perforations in neonates with birth weight less than 1,000 $\mathrm{g}$ not associated with necrotizing enterocolitis. J Pediatr Surg 1991;26(6):714-7.

2. Pumberger W, Mayr M, Kohlhauser C, Weninger M. Spontaneous localized intestinal perforation in very-lowbirth-weight infants: a distinct clinical entity different from necrotizing enterocolitis. J Am Coll Surg 2002;195(6):796803.

3. Best KE, Tennant PW, Addor MC, Bianchi F, et al. Epidemiology of small intestinal atresia in Europe: a register-based study. Arch Dis Child Fetal Neonatal Ed 2012;97(5):F353-8.

4. Tatekawa $Y$, Muraji T, Imai Y, Nishijima E, et al. The mechanism of focal intestinal perforations in neonates with low birth weight. Pediatr Surg Int 1999;15(8):549-52.

5. Jain S, Wadhwa N,MunjalS. Segmental absence of intestinal musculature with intestinal atresia and ileal perforation. Pathology 2009;41(6):596-8.

6. Oretti C, Bussani R, Janes A, Demarini S. Multiple segmental absence of intestinal musculature presenting as spontaneous isolated perforation in an extremely lowbirth-weight infant. J Pediatr Surg 2010;45(8):E25-7.

7. Stephens D, Arensman R, Pillai S, Alagiozian-Angelova V. Congenital absence of intestinal smooth muscle: a case report and review of the literature. I Pediatr Surg 2009;44(11):2211-5.

8. Eicher C, Seitz G, Bevot A, Moll M, et al. Surgical management of extremely low birth weight infants with neonatal bowel perforation: a single-center experience and a review of the literature. Neonatology 2012;101(4):285-92.

9. Moss RL, Dimmitt RA, Barnhart DC, Sylvester KG, et al. Laparotomy versus peritoneal drainage for necrotizing enterocolitis and perforation. $N$ Engl J Med 2006;354(21):2225-34.

10. Blakely ML, Lally KP, McDonald S, Brown RL, et al. Postoperative outcomes of extremely low birth-weight infants with necrotizing enterocolitis or isolated intestinal perforation: a prospective cohort study by the NICHD Neonatal Research Network. Ann Surg 2005;241(6):984-9. 